\title{
Long-term efficacy of bosentan in treatment of pulmonary arterial hypertension in children
}

\author{
A.A. Hislop*,\#, , S. Moledina*, ${ }^{\star}$, H. Foster*, I. Schulze-Neick* and S.G. Haworth*\#
}

ABSTRACT: The aim of the present study was to evaluate a 5-yr experience of bosentan in children with pulmonary arterial hypertension (PAH). A retrospective, observational study was made of children in the UK Pulmonary Hypertension Service for Children (Great Ormond Street Hospital for Children, London, UK) who were given bosentan as monotherapy or in combination, from February 2002 to May 2008 and followed up for $\geqslant 6$ months.

Detailed studies were made of 101 children with idiopathic PAH (IPAH) $(n=42)$ and PAH associated with congenital heart disease $(n=59)$. Before treatment, World Health Organization (WHO) functional class, 6-min walk distance (6MWD), height, weight and haemodynamic data were determined. Evaluations were analysed after 6 months and annually to a maximum of 5 yrs.

Median duration of treatment was $\mathbf{3 1 . 5}$ months. Initial improvement in WHO functional class and 6MWD was maintained for up to 3 yrs. Height and weight increased but the z-scores did not improve. After 3 yrs, bosentan was continued as monotherapy in only $21 \%$ of children with IPAH, but in $69 \%$ of repaired cases and $56 \%$ of those with Eisenmenger syndrome. The Kaplan-Meier survival estimates for the 101 patients were $96,89,83$ and $60 \%$ at 1, 2, 3 and 5 yrs, respectively.

A treatment regime that includes bosentan is safe and appears to be effective in slowing disease progression in children with PAH.

KEYWORDS: Bosentan, idiopathic pulmonary arterial hypertension, paediatric pulmonary arterial hypertension, pulmonary hypertension in congenital heart disease

E ndothelin-1 (ET) is important in the pathobiology of pulmonary arterial hypertension (PAH) [1-3] and plasma levels are elevated in both adults and children with $\mathrm{PAH}$. Its actions are mediated principally by two receptors, $\mathrm{ET}_{\mathrm{A}}$ and $\mathrm{ET}_{\mathrm{B}}$. The dual endothelin receptor antagonist bosentan has been shown to be safe and efficacious in the treatment of adults with PAH $[4,5]$. Recent studies have shown that benefit is generally sustained for $\geqslant 1$ yr [6-9]. In children, evidence for efficacy is limited. In 2003, a small, 12-week trial of 19 children [10] showed that the drug was efficacious and could be used safely in the young. 2 yrs later, ROSENZWEIG et al. [11] reported on 86 children with idiopathic PAH (IPAH) and PAH associated with either congenital heart disease or connective tissue disease. The median duration of exposure to bosentan was 14 months and the drug was considered efficacious, whether given as monotherapy or with a prostanoid. In 2006, we reported on 40 children treated for a mean of 12.7 months by the UK Pulmonary Hypertension Service. In those with IPAH, clinical stability was maintained or achieved, but $60 \%$ of the children also required epoprostenol [12]. Those with associated PAH (APAH) showed a significant improvement in 6min walk distance (6MWD) and World Health Organization (WHO) functional class, the majority of whom were receiving bosentan monotherapy. In the present study, we describe a 5-yr experience of using bosentan both as a monotherapy, and in combination with a prostanoid and/or the phosphodiesterase inhibitor sildenafil in 101 children with IPAH and APAH.

Between February 2002 and May 2008, 101 children with IPAH $(n=42$; two cases familial) or APAH, either post-repair $(n=31)$ or with Eisenbosentan. 32 of these patients, 20 with IPAH and 12 with APAH, had been the subject of our previous report on the short-term efficacy of bosentan [12]. 20 children had trisomy 21 , the majority of these having Eisenmenger syndrome (table 1).

\section{PATIENTS AND METHODS} menger syndrome $(n=28)$, were treated with

\section{AFFILIATIONS}

*Great Ormond Street Hospital for Children, and

\#Institute of Child Health, University

College London, London, UK.

"These authors contributed equally to the manuscript.

CORRESPONDENCE A.A. Hislop UCL Institute of Child Health 30 Guilford Street London WC1N 1EH UK

E-mail: a.hislop@ich.ucl.ac.uk

Received:

April 062010

Accepted after revision: Nov 302010

First published online:

Dec 222010 


\begin{tabular}{|c|c|c|c|c|c|}
\hline $\begin{array}{l}\text { Demographic, clinica } \\
\text { pulmonary arterial hy }\end{array}$ & $\begin{array}{l}\text { ic characteristi } \\
\text { nd pulmonary }\end{array}$ & $\begin{array}{l}\text { start of bose } \\
\text { al hypertensi }\end{array}$ & $\begin{array}{l}\text { treatment ir } \\
\text { sociated wi }\end{array}$ & $\begin{array}{l}\text { Ildren with idic } \\
\text { ongenital hea }\end{array}$ & $\begin{array}{l}\text { athic } \\
\text { disease }\end{array}$ \\
\hline Characteristic & All subjects & IPAH & Repaired & $\begin{array}{l}\text { Eisenmenger } \\
\text { syndrome }\end{array}$ & p-value ${ }^{\#}$ \\
\hline \multicolumn{6}{|l|}{ Demographic } \\
\hline Subjects $n$ & 101 & 42 & 31 & 28 & \\
\hline Trisomy 21 n (\%) & $20(20)$ & $2(5)$ & $3(10)$ & $15(54)$ & $<0.001$ \\
\hline Age at presentation yrs & $8.9 \pm 5.3$ & $7.4 \pm 5.4$ & $8.7 \pm 5.1$ & $11.5 \pm 4.5$ & 0.005 \\
\hline Age starting bosentan yrs & $9.7 \pm 5.5$ & $8.0 \pm 5.7$ & $9.6 \pm 5.0$ & $12.3 \pm 4.7$ & 0.004 \\
\hline Monotherapy n (\%) & $67(67)$ & $24(57)$ & $20(64)$ & $23(82)$ & \\
\hline \multicolumn{6}{|l|}{ Clinical } \\
\hline Weight z-score & $-0.95 \pm 1.8^{\circ}$ & $-0.66 \pm 1.77^{\circ}$ & $-1.71 \pm 1.92^{\circ}$ & $-0.49 \pm 1.42$ & 0.02 \\
\hline Subjects n & 79 & 40 & 21 & 18 & \\
\hline Pra $\mathrm{mmHg}$ & $7.6 \pm 3.4$ & $6.9 \pm 3.5$ & $8.9 \pm 3.4$ & $8.1 \pm 2.2$ & 0.05 \\
\hline $\bar{P}$ pa $\mathrm{mmHg}$ & $56.4 \pm 21.4$ & $60.9 \pm 24.1$ & $45 \pm 17.9$ & $61.3 \pm 11.3$ & 0.003 \\
\hline $\bar{P}$ pa on iNO mmHg & $53.13 \pm 19.5$ & $56.2 \pm 21$ & $42.5 \pm 16.9$ & $60.9 \pm 11.8$ & 0.0066 \\
\hline PVRI U $\mathrm{m}^{2}$ & $21.07 \pm 13.8$ & $22.5 \pm 13.8$ & $14.2 \pm 10.1$ & $28.12 \pm 15.2$ & 0.003 \\
\hline PVRI on iNO U.m² & $17.37 \pm 12.6$ & $17.6 \pm 11.7$ & $11.3 \pm 7.1$ & $27.07 \pm 16.5$ & 0.003 \\
\hline$Q_{p} L \cdot \min \cdot \mathrm{m}^{-2}$ & $2.68 \pm 1.19$ & $2.68 \pm 1.13$ & $2.38 \pm 0.55$ & $2.91 \pm 1.8$ & 0.90 \\
\hline Qp on iNO & $3.36 \pm 2.36$ & $3.46 \pm 2.45$ & $3.4 \pm 2.6$ & $2.94 \pm 1.93$ & 0.62 \\
\hline
\end{tabular}

In all patients, the investigations confirmed the presence of PAH and determined its causality. An ECG, transthoracic echocardiogram, chest radiograph and, where indicated, computed chest tomography were carried out at presentation. Cardiac catheterisation was carried out under general anaesthesia. The pulmonary blood flow was calculated based on measured oxygen consumption in the majority of cases and acute vasodilator testing was carried out using inhaled nitric oxide. Baseline haemodynamic data were recorded (table 1).

The treatment algorithm used was based on the European Society of Cardiology algorithm for the management of adult patients with IPAH and the national guidelines [13, 14], which were adapted for children. The few children with a positive response to acute vasodilator testing with nitric oxide at cardiac catheterisation were given a calcium channel antagonist, nifedipine. For the nonresponders, in IPAH and post-operative pulmonary hypertension, all functional class IV patients were treated with epoprostenol at presentation, as were the severely symptomatic children in functional class III. Less symptomatic children in class III were treated with bosentan either as monotherapy or in combination with sildenafil, with escalation of therapy if they failed to demonstrate a response. Children with unrepaired congenital heart disease received bosentan or bosentan was added to the sildenafil they were receiving before referral to the Service. Those with IPAH and post-operative pulmonary hypertension were anticoagulated with warfarin, but the younger children were given aspirin. The therapeutic regimen was tailored to the needs of each child and adjusted according to response. The target dose of bosentan was $31.5-125 \mathrm{mg}$ b.i.d. according to weight $[11,12]$. Children $<10 \mathrm{~kg}$ in weight were given $15 \mathrm{mg}$ b.i.d. If patients deteriorated on bosentan monotherapy then sildenafil or epoprostenol were added as required. All children were known to have normal liver function and haematological studies before bosentan was prescribed.

It should be noted that management changed as new drugs became available during the course of the study period, from early 2002.

Of the 101 cases with IPAH and congenital heart disease, bosentan was given to 67 children as monotherapy ( 24 with IPAH and 43 APAH). 58 of these children had not been treated previously (treatment naïve) and nine had their medication changed to bosentan. In 34 patients (18 IPAH and $16 \mathrm{APAH}$ ), bosentan was added to an existing pulmonary hypertension-specific therapy, either sildenafil or a prostanoid, or both. Immediately before starting treatment, the weight, height and WHO functional class were determined. The 6MWD was measured in those children old enough and developmentally able to carry out the test successfully $(n=51)$. In 59 out of the 101 children ( 24 with IPAH, 16 with repaired defects and 19 with Eisenmenger syndrome), the height, weight, WHO functional class and 6MWD had also been recorded $\sim 6$ months (mean \pm SD $4.9 \pm 2.8$ months) before being started on bosentan, and 23 (39\%) of these children were already on alternative specific treatment for pulmonary hypertension. 
a)

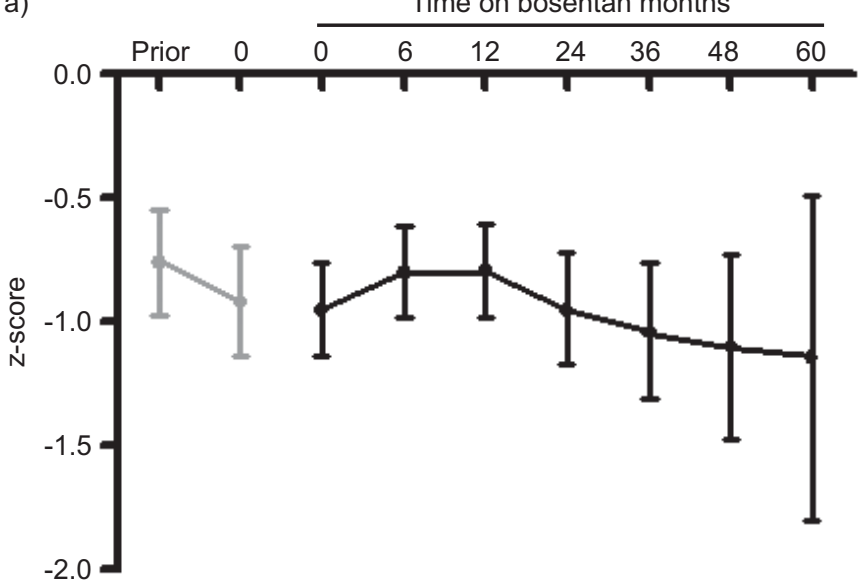

b)

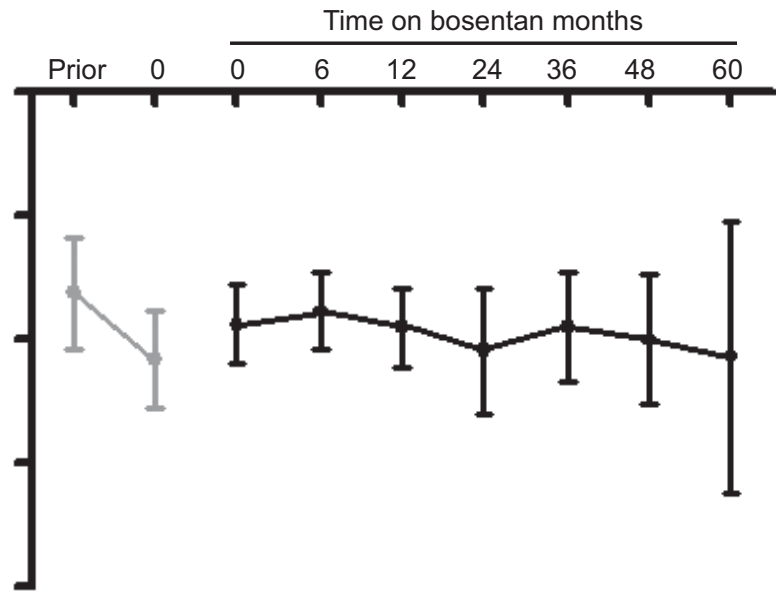

FIGURE 1. Mean a) weight and b) height z-scores throughout the study period, showing no significant change with treatment. Values for the cases assessed prior to the start of treatment (time 0 ) are shown in grey.

In all children, the weight, height, WHO functional class and 6MWD were assessed at 6 months (6.3 \pm 1.4 months), $1 \mathrm{yr}$ (12.2 \pm 1.5 months), 2 yrs (23.9 \pm 2.02 months), 3 yrs (35.5 \pm 1.8 months $), 4$ yrs $(47.9 \pm 1.7$ months $)$ and 5 yrs $(60.3 \pm 1.8$ months) after starting treatment with bosentan. Liver function tests were carried out at monthly intervals. 19 children had a second cardiac catheterisation study. Initiation of additional therapies and discontinuation of bosentan was noted.

\section{Statistical methods}

Data are presented as mean $\pm S D$ or median (interquartile range), as appropriate. Height and weight are expressed as standard deviation scores (z-scores) based on data from normal British children [15] or data specific for children with Down's syndrome [16]. 6MWD is expressed as \% predicted based on a published regression equation for children [17].

The Kruskal-Wallis test was used to compare variables between diagnostic groups. Comparison of height z-score, weight z-score and 6MWD between baseline and follow-up visits was performed using the paired t-test. The Wilcoxon matched paired test was used to test the significance of changes in WHO functional class over the appropriate time intervals. Survival, assessed from starting bosentan to death or the end of the study, was summarised using Kaplan-Meier estimates. Patients were censored at transplantation, discontinuation of bosentan or the end of follow-up. Treatment failure in the monotherapy group was defined as time from starting bosentan to discontinuation of bosentan for any reason, initiation of additional medical therapy, transplantation or death. Statistical analysis was performed using GraphPad Prism version 4.0 (GraphPad Software Inc., San Diego, CA, USA). This work was approved by the ethics committee of Great Ormond Street Hospital and University College London Institute of Child Health (London, UK).

\section{RESULTS}

\section{Baseline characteristics}

Findings at the start of bosentan treatment (baseline) are shown in table 1 . The mean \pm SD age at the start of treatment was $9.7 \pm 5.5$ yrs. The youngest child treated was 1 month old.
Females predominated, with the sex ratio being 1.63:1 in IPAH and $1.35: 1$ in APAH.

Both height and weight were below normal (table 1 and fig. 1). The children with repaired congenital heart disease (CHD) were of particularly low weight. The mean baseline WHO functional class was 2.8 (fig. 2), and both the mean and class distribution was similar in the different subgroups (table 1). 51 children were able to perform a 6-min walk test reliably. Their mean age was 11.7 yrs. The mean $\pm \mathrm{SD}$ distance walked was $258 \pm 127 \mathrm{~m}$ ( $40.7 \%$ pred for age) (fig. 3$)$. There was no significant difference between the different diagnostic groups. Comparing children with and without Down's syndrome, there was no difference at baseline in the \% pred 6MWD and WHO functional class.

79 children had a cardiac catheterisation study (table 1). The pulmonary artery pressure and pulmonary vascular resistance were significantly higher in those with IPAH or Eisenmenger syndrome than in the repaired CHD cases.

\section{Clinical change prior to initiation of bosentan therapy}

The 59 children (mean \pm SD age $10.3 \pm 5.4 \mathrm{yrs}$ ) assessed $\sim 6$ months (mean 4.9 months) prior to starting bosentan therapy had deteriorated during this time interval. They had failed to thrive. The $\mathrm{z}$-score for height deteriorated from $-0.81 \pm 1.65$ to $-1.08 \pm 1.4(p=0.08)$ and the score for weight fell from $-0.76 \pm 1.6$ to $-0.93 \pm 1.7(\mathrm{p}=0.02)$ (fig. 1$)$. The mean $\mathrm{WHO}$ score had deteriorated from 2.5 to 2.8 by the time bosentan therapy was started ( $\mathrm{p}=0.04$ ) (fig. 2). The 6MWD was recorded before treatment in 15 of these children and, at 39\% pred, had not changed significantly by the time bosentan was first given (fig. 3).

\section{Clinical response to therapy}

The median duration of bosentan treatment was 31.5 months (range 6-73 months). There were no serious side-effects. The liver function tests became abnormal in three children, necessitating dose reduction in one, temporary suspension of treatment for 1 month in one and replacement by sildenafil in the third child. 

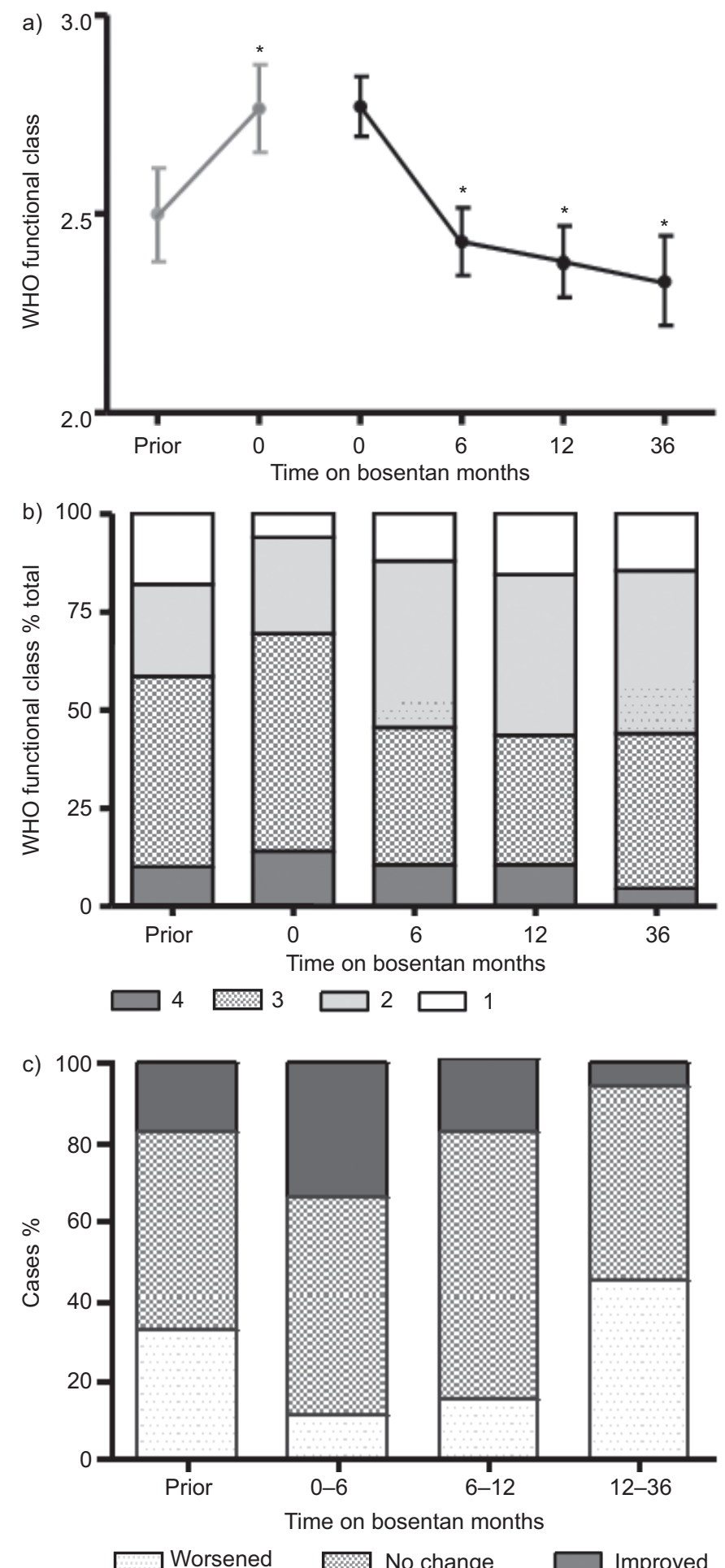

FIGURE 2. a) World Health Organization (WHO) functional class $\sim 6$ months prior to instituting bosentan therapy, at the start of bosentan treatment (time 0 ), and after $0,6,12$ and 36 months. Values for the cases assessed prior to the start of treatment are shown in grey. Data are presented as mean $\pm \mathrm{SD}$. b) The percentage of cases in each WHO functional class. c) The percentage of cases improving, worsening or staying the same with time. ${ }^{*}: p<0.05$.

The height and weight z-scores did not change significantly during follow-up, indicating no further deterioration in failure to thrive but no significant catch-up growth (fig. 1). WHO functional class improved after 6 months treatment, decreasing from a mean of 2.8 to $2.4 \pm 0.8(\mathrm{p}<0.001)$. In the survivors, improvement was maintained for up to 3 yrs $(n=48)$ (fig. 2$)$. All three subgroups showed an improvement in functional class and a similar pattern of response was seen whether bosentan was given as a monotherapy or as an add-on combined therapy.

Exercise tolerance also improved. The 6MWD increased from a baseline of $40.9 \%$ pred ( $258 \mathrm{~m} ; \mathrm{n}=51)$ to $49.3 \%$ pred $(312 \mathrm{~m})$ at 6 months $(p<0.01)$, an increase maintained at 1,2 and 3 yrs $(\mathrm{p}<0.01$ for all years; $n=51, n=48$ and $n=39$, respectively) (fig. 3a). 6MWD increased from 42.9 to $58.6 \%$ pred $(\mathrm{p}<0.01)$ at 6 months in those with IPAH, while the repaired cases showed a significant increase to $57 \%$ pred, but only after 1 yr $(p<0.01)$ (fig. 3b). Although there was no significant difference between the three subgroups at the start of bosentan treatment, those with Eisenmenger syndrome improved less than those in the other two groups combined at 6 months and 1 yr $(\mathrm{p}<0.05)$.

In the entire cohort, there was no significant difference between those with or without Down's syndrome in the response to treatment, or specifically within the group of children with Eisenmenger syndrome, which had the majority of patients with Down's syndrome.

On repeat cardiac catheterisation $(n=19)$, after a median of 17 months (range 8-33 months), there was no significant change in either mean \pm SD pulmonary artery pressure $(48.8 \pm$ 16.6 versus $48.3 \pm 16.2 \mathrm{mmHg}$ ) or pulmonary vascular resistance index $\left(16.5 \pm 2.6\right.$ versus $\left.14.1 \pm 2.0 \mathrm{U} \cdot \mathrm{m}^{2}\right)$. 15 of these patients were on bosentan as monotherapy, and catheterisation was carried out to confirm that they remained stable and no intensification of therapy was needed. They were indeed found to be stable.

In the subgroup of 59 children who had been assessed $\sim 5$ months prior to treatment with bosentan, after 6 months of treatment, there was a small but nonsignificant increase in $\mathrm{z}$-score for height and weight, an increase in 6MWD and a decrease in WHO functional class. Their response to bosentan was not as great as that of the group as a whole (fig. 4). However, the survival of these 59 prior patients was not significantly different to the other cases (94.8, 90.8, 85.9 and $60.2 \%$ versus $97.6,86.8,79.6$ and $74.3 \%$ at $1,2,3$ and 5 yrs, respectively; $\mathrm{p}=0.83$ ).

\section{Changes in treatment}

Bosentan was discontinued in seven children. One child had a persistently raised alanine transaminase level to more than three times the upper limit of normal, even when the bosentan dose was decreased, but became normal when the drug was discontinued. One child with Eisenmenger syndrome had mild systemic hypotension, one was successfully weaned off the drug and four repeatedly refused to have liver function tests carried out.

\section{Escalation of therapy}

Of the 24 children with IPAH started on monotherapy, 14 $(58 \%)$ required additional therapy for worsening of symptoms (fig. 5a). Sildenafil was added in seven children, intravenous epoprostenol in six children and both drugs in one child. Of the 43 cases with APAH who were started on monotherapy, 

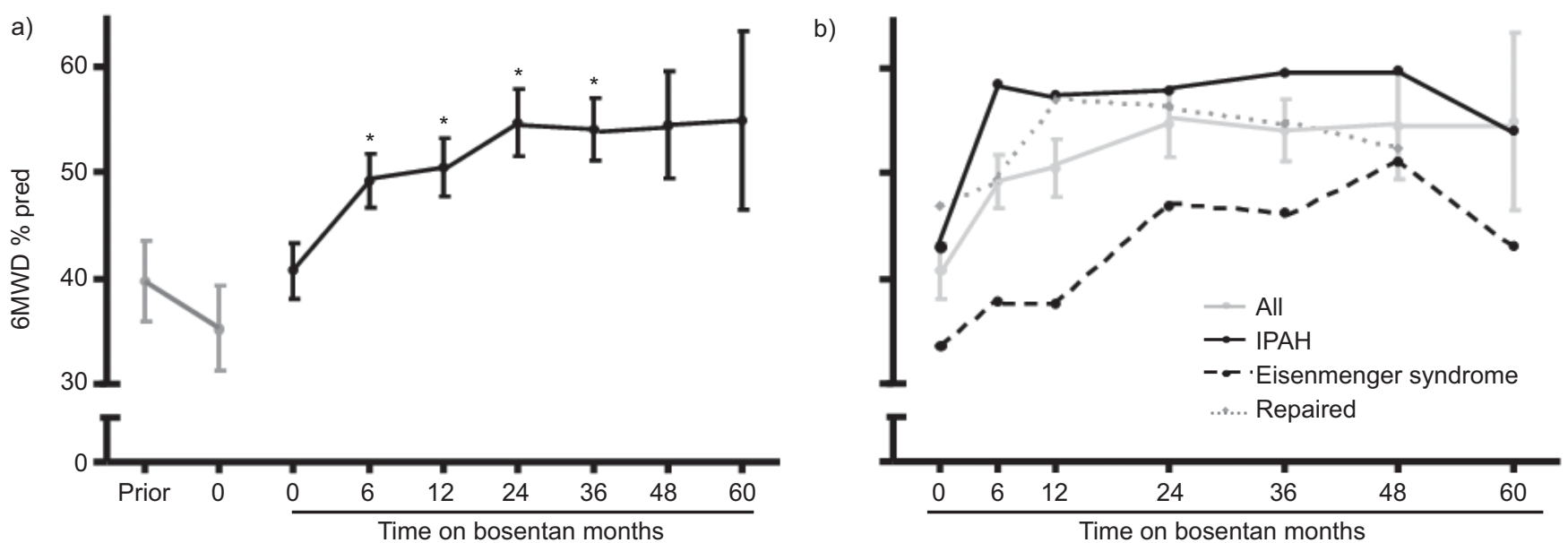

FIGURE 3. a) 6-min walk distance (6MWD). \% predicted (\% pred) for age prior to starting treatment and during treatment with bosentan. Values for the cases assessed prior to and at the start of treatment (time 0 ) are shown in grey. b) 6MWD \% pred by diagnostic group. IPAH: idopathic pulmonary arterial hypertension. *: $p<0.05$.

16 (37\%) needed an additional medication (fig. 5a). Sildenafil was added in 12 children, epoprostenol in two and both drugs in two cases.

In those children in whom bosentan was added to an existing medication, no further changes in treatment were made. Seven children, all with IPAH and on combination therapy, had a bilateral lung or heart-lung transplant, having been on bosentan for a mean time of 18.4 months. All were still alive a mean of 37 months later. Thus, over the course of followup, bosentan was discontinued in seven patients, additional therapy was given to 30 , seven patients underwent transplantation and 21 patients died. Those in whom bosentan was discontinued were still alive at the end of the study period.

\section{Survival}

For the 101 patients, the Kaplan-Meier survival estimates were $95,89,83$ and $60 \%$ at $1,2,3$ and 5 yrs respectively. Eight children with IPAH and 13 children with APAH died. The survival values for IPAH were 95, 95, 95 and 55\%, for the repaired cases were 97, 86, 78 and $58 \%$, and for Eisenmenger syndrome were $92,84,74$ and $74 \%$, at $1,2,3$ and 5 yrs, respectively, (fig. 5b) with no significant difference between the groups. The data for transplant-free survival at 1, 2, 3 and 5 yrs in the IPAH group were 94, 83, 75 and 56\%.

\section{DISCUSSION}

Our study shows that a treatment regime that includes bosentan is safe and efficacious in the long-term management of children with PAH. We have extended the findings of earlier studies $[11,12]$ by showing that bosentan is efficacious for a median time of 31.5 months in a large cohort of children with PAH. We previously reported on 40 children, those with IPAH showed stabilisation after a mean follow-up of 12.7 months on bosentan, while those with APAH showed a significant improvement in New York Heart Association (NYHA) functional class, 6MWD and weight [12]. In the current series of 101 children with IPAH and APAH as a result of congenital heart disease, improvement was seen within 6 months and could be maintained for up to 3 yrs, giving bosentan either as monotherapy or in combination with other specific therapies. 10 children were successfully maintained on bosentan for 5 yrs, with half receiving the drug as a monotherapy. The drug appeared safe, in that the aspartate transaminase levels became persistently elevated in only one child, confirming earlier paediatric studies [11, 12]. Recent post-market surveillance reported elevated transaminases in $2.7 \%$ of children, compared with $7.8 \%$ in patients $>12$ yrs of age [18]. Young age should not be a deterrent to treatment with bosentan. The youngest child treated in the present study was 1 month old. Our experience confirms that of others [19]. In addition, a new paediatric formulation has just been trialled successfully and the formulation has been approved, easing the management of infants and young children [20].

Bosentan is used most widely in both children and adults with IPAH and congenital heart disease, and we focussed on these diagnostic groups in the present paper. The median follow-up for these 101 children was 31.5 months, comparing favourably with a mean follow-up of 24 months in an adult cohort with IPAH [8] and a median of 29 months in adults with Eisenmenger syndrome [9]. In the present study, the 1- and 2-yr survival for IPAH and APAH associated with congenital heart disease combined was 95 and $89 \%$ respectively, with no significant difference in survival between the diagnostic groups or between the Down's syndrome and normal children. These figures compare favourably with the only other comparable paediatric study, which showed a 2-yr survival of $91 \%$ [11]. In adults with IPAH, the survival of patients treated with bosentan as a first-line therapy with the addition of other medicines as indicated was reported as 92 and $89 \%$ at 1 and 2 yrs in a European study [8], and 96 and 89\%, respectively, in an American study [21]. In a recent detailed study of IPAH in children, we found no difference in survival between incident and prevalent cases [22].

We had data on 59 children who had been assessed $\sim 5$ months before treatment with bosentan was started. In the interim, these children deteriorated rapidly, as judged by their height, weight and WHO functional class. The need for prompt referral to a pulmonary hypertension specialist centre is selfevident, even when the symptomatology is mild. Early treatment has been shown to be beneficial in adults [23]. In 
a)
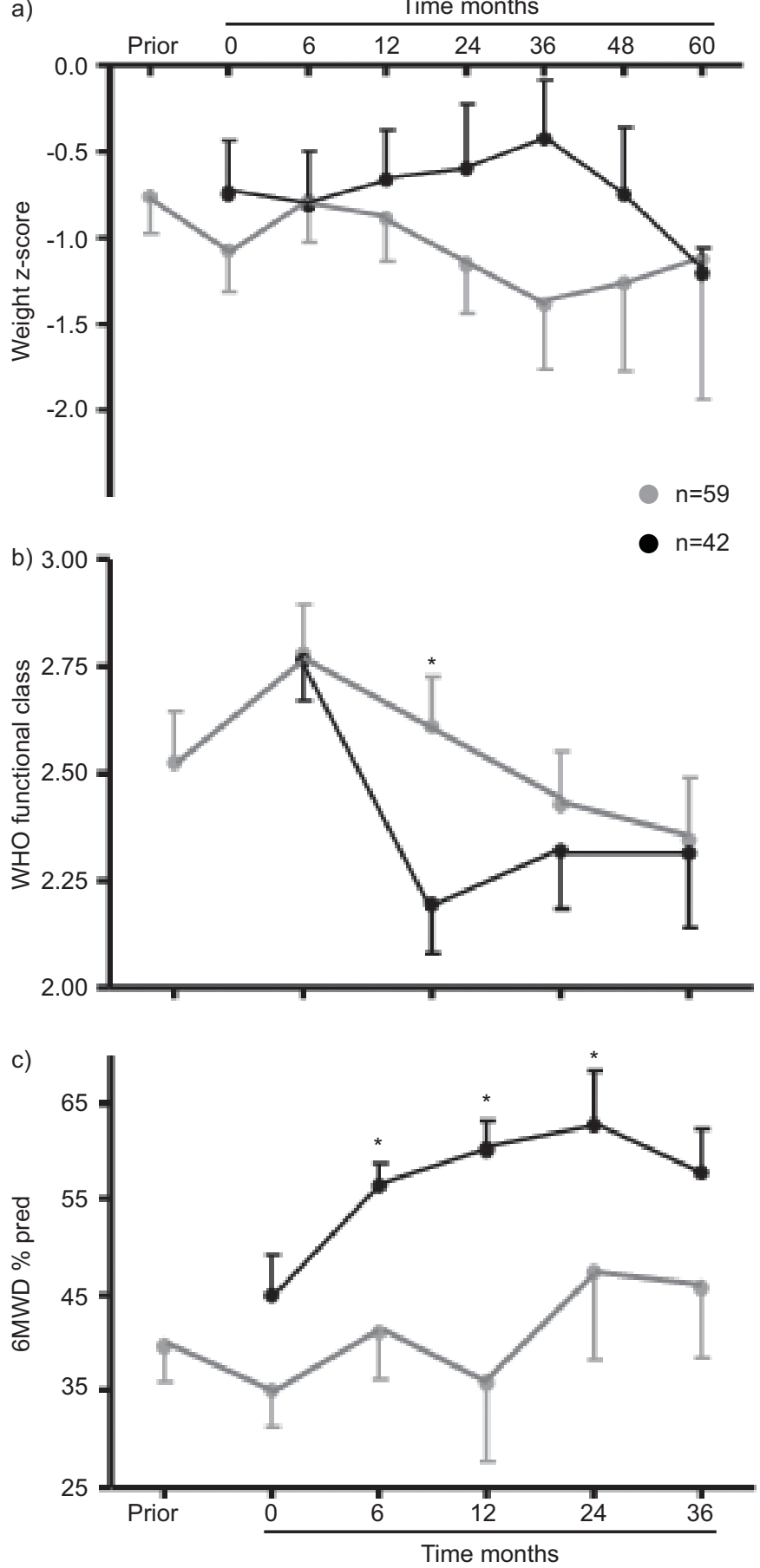

FIGURE 4. Comparison of the cases studied 4.9 months before starting bosentan $(n=59)$ and the remaining cases $(n=42)$ for a) weight centile, b) World Health Organization (WHO) functional class and c) 6-min walk distance (6MWD) \% predicted (\% pred) at start of bosentan therapy and during follow-up. *: $p<0.05$.

these 59 paediatric cases, bosentan restored the clinical status to what it had been when the children first presented almost 5 months earlier, whether given as monotherapy or added to an existing therapy. However, they did not catch up. Their values for weight, WHO functional class and 6MWD during follow-up were all less than in children treated more promptly, although each parameter was not significantly poorer and the survival figures were not significantly different $(p=0.83)$. This mirrors the findings of the open-label extension of the Bonsentan Ramdomized Trial of Endothelin Antagonist Therapy (BREATHE)-5 study on adults with Eisenmenger syndrome that those who had previously been in the placebo arm did not increase their 6MWD after 24 weeks medication with bosentan as much as patients treated with bosentan from the outset [24]. Assessing the efficacy of medicines is difficult in pulmonary hypertension, particularly in children. A positive effect is conventionally accepted as an improvement in exercise capacity (generally the 6MWD in adults and older children), WHO functional class, absence of need to give additional therapies or to transplant, and improved survival. We found that both WHO functional class and 6MWD improved within 6 months of starting treatment, and improvement was maintained for at least 3 yrs, which is longer than previously reported. In adults with IPAH, an improvement in 6MWD was seen at 4 months and maintained at 1 yr [8], while improvement was seen for $\leqslant 2$ yrs in those with Eisenmenger syndrome and other types of congenital heart disease [9]. A small study reported that an improvement in 6MWD seen after 4 months treatment was maintained in adults but not children with either Eisenmenger syndrome or post-operative $\mathrm{PAH}$ [25]. However, other investigators found that bosentan improved or stabilised NYHA functional class in children $[11,18,26]$. Growth may be an additional marker of treatment efficacy in children [22]. Using these criteria, we found that the children continued to grow but there was no change in the z-scores for height and weight. Relatively few children were recatheterised but those who were re-studied did not show a change in haemodynamics. Others have reported an improvement, although the absolute changes in pressure and resistance have usually been modest in both children and adults $[8,10,11]$. Given that pulmonary vascular disease is a progressive disease, it is reassuring to find that the patients did not show a deterioration in haemodynamics.

In the course of the present study, 39\% of all children started on bosentan monotherapy needed additional sildenafil or epoprostenol, or both. About $65 \%$ of children with IPAH remained event-free at $1 \mathrm{yr}$ and $38 \%$ at 2 yrs, without additional therapy for worsening of symptoms, transplantation or death. The median time to treatment failure was 18 months. An adult study found that 61 and $44 \%$ of patients had not required either prostanoid therapy or transplantation at 1 and 2 yrs, respectively [8], figures not markedly different to our own in children. As expected, the children with congenital heart disease fared better than those with IPAH: only $37 \%$ of those on bosentan monotherapy needed additional treatment and the median time to treatment failure was longer (39.2 and 54.2 months for those with Eisenmenger syndrome and postrepair pulmonary hypertension, respectively). Thus, although survival in the three diagnostic groups was similar, the children with pulmonary hypertension associated with congenital heart disease needed less additional therapy to maintain improvement than those with IPAH.

In summary, these studies indicate that bosentan alone or with other pulmonary hypertensive treatment can be effective in the long-term management of children with PAH. For at least the first 3 yrs, the results of treatment are at least as good as those in adults, and the children have been followed up for longer 

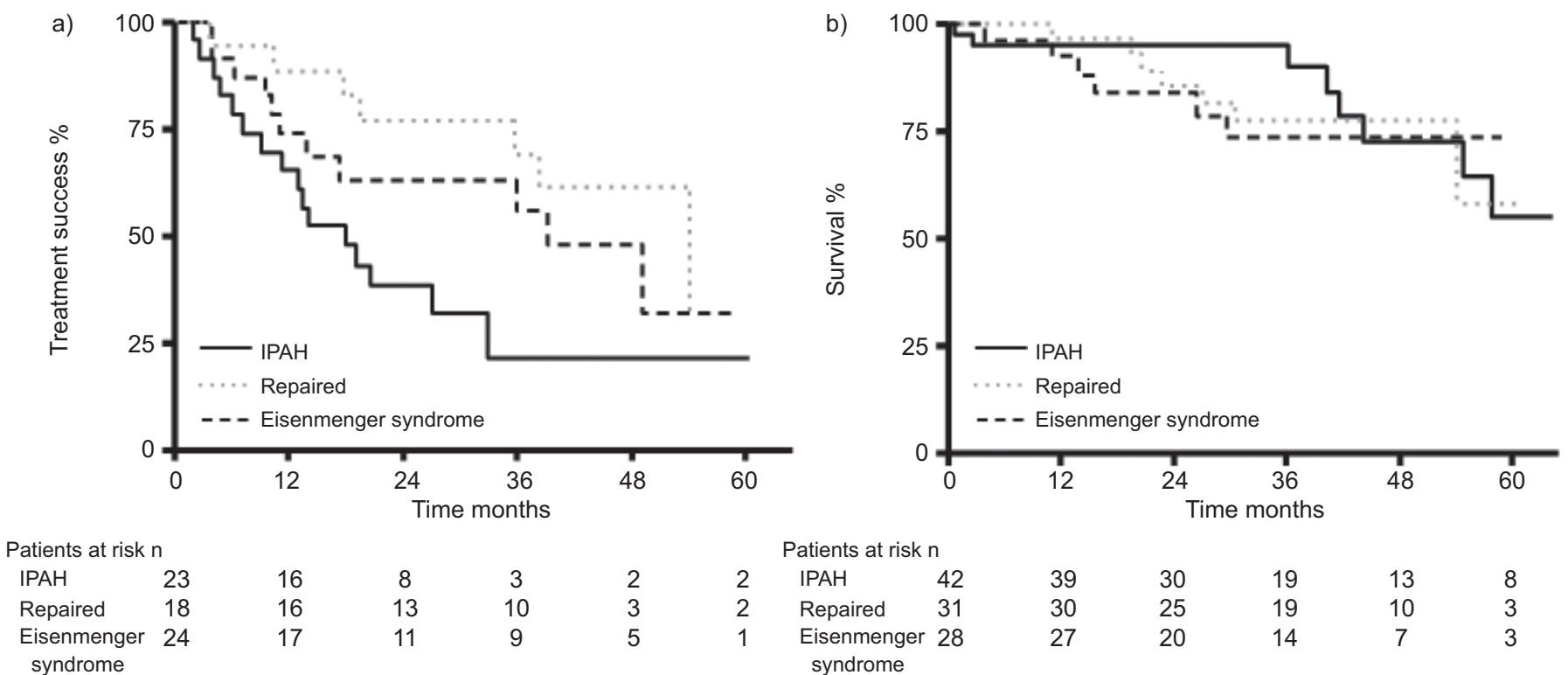

16
16
17

$\begin{array}{cc}8 & 3 \\ 13 & 10 \\ 11 & 9\end{array}$

2
3
5

\begin{tabular}{llr}
\multicolumn{4}{c}{ Patients at risk n } \\
2 & IPAH & 42 \\
2 & Repaired & 31 \\
1 & Eisenmenger & 28 \\
& syndrome
\end{tabular}

$\begin{array}{ccccc}39 & 30 & 19 & 13 & 8 \\ 30 & 25 & 19 & 10 & 3 \\ 27 & 20 & 14 & 7 & 3\end{array}$

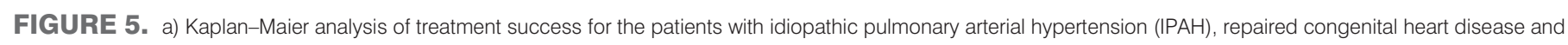

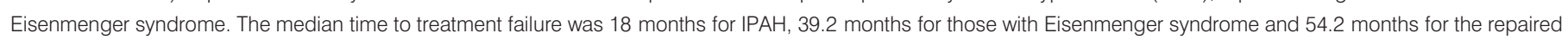
cases ( $p=0.03$, log rank test for trend). b) Survival for these three groups of patients, with transplantation as a censoring event.

( $\leqslant 5$ yrs) [8]. Side-effects are unusual, but monthly liver function tests remain mandatory. Pulmonary vascular disease is, however, a progressive disease, and since the majority of our patients with IPAH and a significant proportion of those with APAH needed additional medication to sustain improvement, these children need to be followed up very closely indeed. The number of children followed up for $5 \mathrm{yrs}$ is relatively small, but it does appear that in IPAH, survival falls significantly after 3 yrs of treatment, despite escalation of therapy. This emphasises the need for a proactive, aggressive approach to the management of IPAH in children with combination therapy, treating the three signalling pathways known to be involved in the pathobiology of pulmonary vascular disease.

Based on our experience we would recommend: 1) close follow-up supervision; and 2) starting combination therapy at presentation. In patients with IPAH, dual oral therapy can be used if the patient is in functional class I or II, or relatively well in class III. All class IV and severely symptomatic class III children should receive intravenous epoprostenol, and ideally bosentan and sildenafil. In a less critical situation, the family usually needs a few weeks on combined oral therapy to begin to adjust to having a very sick child whom they had previously thought normal, before starting intravenous therapy. Quality of life, for the child and the family, is extremely important, particularly when the disease can not be cured.

We need new drugs that target deranged signalling pathways. This will entail designing clinical trials specifically for children to determine safety, efficacy and optimum dosing criteria. The need for long-term follow-up to detect any detrimental effect on the growing child is self-evident, and applies to all existing and new drugs used to treat pulmonary hypertension in children.

\section{STATEMENT OF INTEREST}

Statements of interest for all authors can be found at www.erj. ersjournals.com/site/misc/statements.xhtml

\section{ACKNOWLEDGEMENTS}

We thank the physicians who referred children to the UK Pulmonary Hypertension Service for Children (Great Ormond Street Hospital for Children, London, UK) and helped care for them. We also thank Y. Flynn (UK Pulmonary Hypertensive Service), our clinical specialist nurse, for all her support.

\section{REFERENCES}

1 MacLean MR. Endothelin-1: a mediator of pulmonary hypertension? Pulm Pharmacol Ther 1998; 11: 125-132.

2 Allen SW, Chatfield BA, Koppenhafer SA, et al. Circulating immunoreactive endothelin-1 in children with pulmonary hypertension. Association with acute hypoxic pulmonary vasoreactivity. Am Rev Respir Dis 1993; 148: 519-522.

3 Giaid A, Yanagisawa M, Langleben D, et al. Expression of endothelin-1 in the lungs of patients with pulmonary hypertension. N Engl J Med 1993; 328: 1732-1739.

4 Chen SJ, Chen YF, Meng QC, et al. Endothelin-receptor antagonist bosentan prevents and reverses hypoxic pulmonary hypertension in rats. J Appl Physiol 1995; 79: 2122-2131.

5 Holm P, Liska J, Clozel M, et al. The endothelin antagonist bosentan: hemodynamic effects during normoxia and hypoxic pulmonary hypertension in pigs. J Thorac Cardiovasc Surg 1996; 112: 890-897.

6 Channick RN, Simonneau G, Sitbon O, et al. Effects of the dual endothelin-receptor antagonist bosentan in patients with pulmonary hypertension: a randomised placebo-controlled study. Lancet 2001; 358: 1119-1123.

7 Sitbon O, Badesch DB, Channick RN, et al. Effects of the dual endothelin receptor antagonist bosentan in patients with pulmonary arterial hypertension: a 1-year follow-up study. Chest 2003; 124: $247-254$. 
8 Provencher S, Sitbon O, Humbert M, et al. Long-term outcome with first-line bosentan therapy in idiopathic pulmonary arterial hypertension. Eur Heart J 2006; 27: 589-595.

9 Diller GP, Dimopoulos K, Kaya MG, et al. Long-term safety, tolerability and efficacy of bosentan in adults with pulmonary arterial hypertension associated with congenital heart disease. Heart 2007; 93: 974-976.

10 Barst RJ, Ivy D, Dingemanse J, et al. Pharmacokinetics, safety, and efficacy of bosentan in pediatric patients with pulmonary arterial hypertension. Clin Pharmacol Ther 2003; 73: 372-382.

11 Rosenzweig EB, Ivy DD, Widlitz A, et al. Effects of long-term bosentan in children with pulmonary arterial hypertension. J Am Coll Cardiol 2005 16, 46: 697-704.

12 Maiya S, Hislop AA, Flynn Y, et al. Response to bosentan in children with pulmonary hypertension. Heart 2006; 92: 664-670.

13 Galie N, Torbicki A, Barst R, et al. Guidelines on diagnosis and treatment of pulmonary arterial hypertension. The Task Force on Diagnosis and Treatment of Pulmonary Arterial Hypertension of the European Society of Cardiology. Eur Heart J 2004; 25: 2243-2278.

14 Recommendations on the management of pulmonary hypertension in clinical practice. Heart 2001; 86: Suppl. 1, I1-I13.

15 Freeman JV, Cole TJ, Chinn S, et al. Cross sectional stature and weight reference curves for the UK, 1990. Arch Dis Child 1995; 73: 17-24.

16 Styles ME, Cole TJ, Dennis J, et al. New cross sectional stature, weight, and head circumference references for Down's syndrome in the UK and Republic of Ireland. Arch Dis Child 2002; 87: 104-108.

17 Geiger R, Strasak A, Treml B, et al. Six-minute walk test in children and adolescents. J Pediatr 2007; 150: 395-399.

18 Beghetti M, Hoeper MM, Kiely DG, et al. Safety experience with bosentan in 146 children 2-11 years old with pulmonary arterial hypertension: results from the European Postmarketing Surveillance program. Pediatr Res 2008; 64: 200-204.

19 Gilbert N, Luther YC, Miera O, et al. Initial experience with bosentan (Tracleer) as treatment for pulmonary arterial hypertension $(\mathrm{PAH})$ due to congenital heart disease in infants and young children. Z Kardiol 2005; 94: 570-574.

20 Beghetti M, Haworth SG, Bonnet D, et al. Pharmacokinetic and clinical profile of a novel formulation of bosentan in children with pulmonary arterial hypertension: the FUTURE-1 study. Br J Clin Pharmacol 2009; 68: 948-955.

21 McLaughlin VV. Survival in patients with pulmonary arterial hypertension treated with first-line bosentan. Eur J Clin Invest 2006; 36: Suppl. 3, 10-15.

22 Moledina S, Hislop AA, Foster $\mathrm{H}$, et al. Childhood idiopathic pulmonary arterial hypertension - a national cohort study. Heart 2010; 96: 1401-1406.

23 Galie N, Rubin L, Hoeper M, et al. Treatment of patients with mildly symptomatic pulmonary arterial hypertension with bosen$\tan$ (EARLY study): a double-blind, randomised controlled trial. Lancet 2008; 371: 2093-2100.

24 Gatzoulis MA, Beghetti M, Galie N, et al. Longer-term bosentan therapy improves functional capacity in Eisenmenger syndrome: results of the BREATHE-5 open-label extension study. Int J Cardiol 2008 23, 127: 27-32.

25 van Loon RL, Hoendermis ES, Duffels MG, et al. Long-term effect of bosentan in adults versus children with pulmonary arterial hypertension associated with systemic-to-pulmonary shunt: does the beneficial effect persist? Am Heart J 2007; 154: 776-782.

26 Fasnacht MS, Tolsa JF, Beghetti M. The Swiss registry for pulmonary arterial hypertension: the paediatric experience. Swiss Med Wkly 2007; 137: 510-513. 